Published in final edited form as:

Keshavarzi, B., Javadi, A., Bahramian, A., \& Miller, R. (2018). Thixotropic Bulk Elasticity versus Interfacial Elasticity In Xanthan Gum Surfactant Mixed Solutions. Colloids and Surfaces A:

Physicochemical and Engineering Aspects, 557, 123-130. doi:10.1016/j.colsurfa.2018.01.053.

\title{
Thixotropic Bulk Elasticity versus Interfacial Elasticity In Xanthan Gum Surfactant Mixed Solutions
}

Behnam Keshavarzi, Aliyar Javdi, Alireza Bahramian, Reinhard Miller

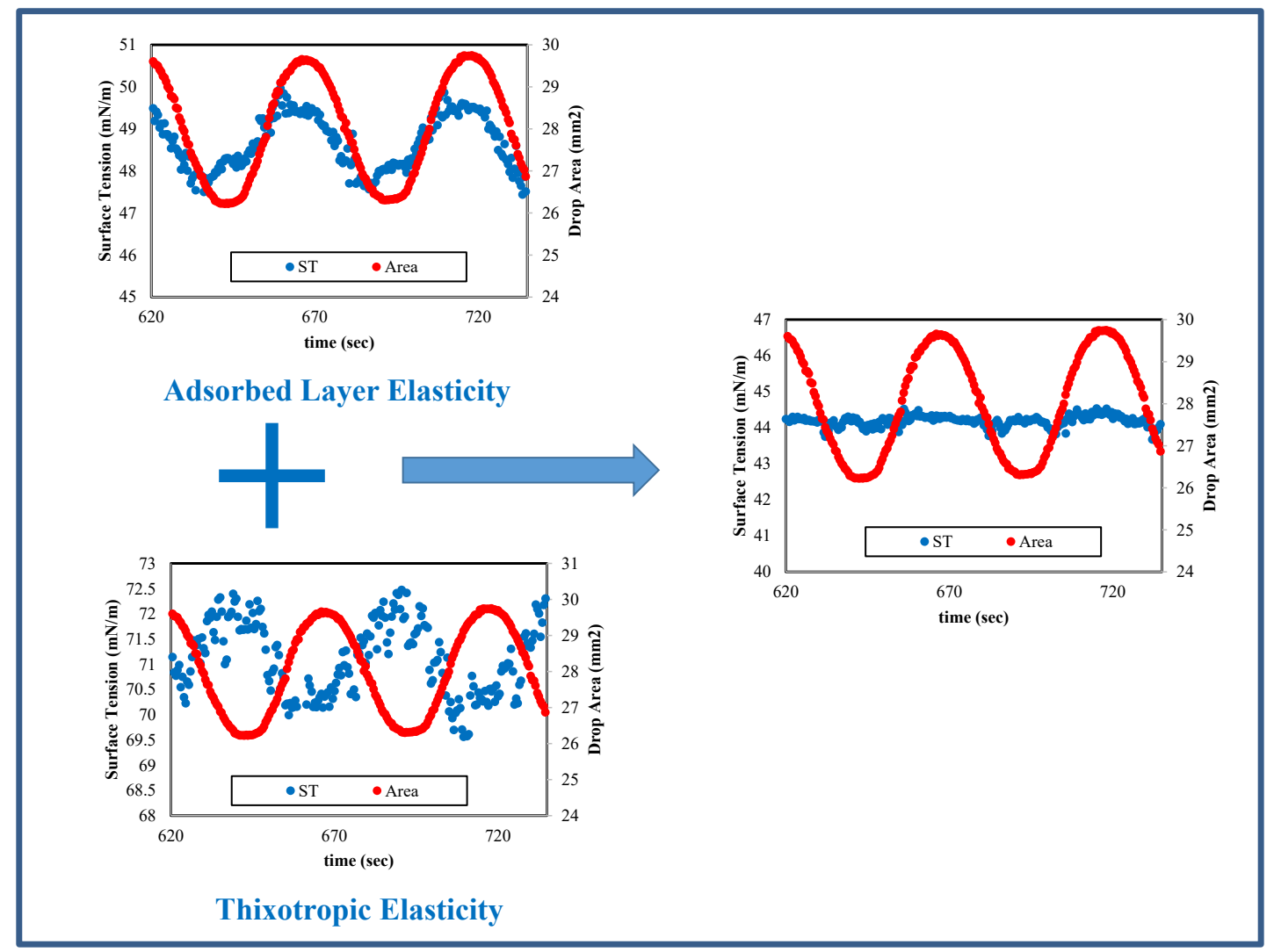

Keywords: Xanthan Gum, Thixotropic fluids, Sodium dodecyl sulfate, Triton x100, Dynamic surface tension, Polymer surfactants interaction. 


\section{Thixotropic Bulk Elasticity versus Interfacial Elasticity}

\section{In Xanthan Gum Surfactant Mixed Solutions}

Behnam Keshavarzi ${ }^{1}$, Aliyar Javdi ${ }^{1,2 *}$, Alireza Bahramian ${ }^{1}$ and Reinhard Miller ${ }^{2}$

${ }^{1}$ Institute of Petroleum Engineering, Chemical Engineering Department, College of Engineering, University of Tehran, Iran

${ }^{2}$ Max Planck Institute of Colloids and Interfaces, Potsdam, Germany

* Corresponding author, email: javadi.aliyar@ut.ac.ir, Institute of Petroleum Engineering, University of Tehran, Iran. 


\begin{abstract}
:
Dynamic surface behavior and interfacial rheology of Xanthan Gum (XG) polymer and surfactant mixed aqueous solutions have been investigated in this work using Profile Analysis Tensiometry (PAT). The interactions of XG with anionic sodium dodecyl sulfate (SDS) and nonionic triton X100 surfactants are studied. The results demonstrate a significant influence of the XG thixotropic bulk rheology behavior on dynamic surface tension measurements. The time dependent thixotropic force (due to the movement and rearrangement of XG molecules under applied shear and relaxation time afterwards) was evaluated using a high precision buoyancy force measurement system. This extra thixotropic force can affect the expected balance of gravity and surface forces in the Gauss-Laplace equations and causes an abnormal increasing dynamic surface tension for aqueous XG solutions versus time in absence of surfactants. It is shown that the thixotropic property of this polymer can also induce an additional surface elasticity which can be measured by drop oscillation experiments via PAT. Therefore, surface elasticity measurements can be proposed as a novel method for determining the extent of fluid thixotropy. The results also showed strong interaction between XG and SDS molecules. At SDS concentrations below critical micelle concentration $(\mathrm{CMC})$, the equilibrium surface tension of XG-SDS solution decreased considerably with increasing XG concentration. This interaction originates from the anionic nature of both XG and SDS molecules. The surface tension behavior of mixed solutions of XG and Triton X100 molecules did not show significant interactions. The obtained results allow improving the ability of the Gauss-Laplace equation to calculate more accurately the dynamic surface tension of thixotropic fluids.
\end{abstract}

Keywords: Xanthan Gum, Thixotropic fluids, Sodium dodecyl sulfate, Triton x100, Dynamic surface tension, Polymer surfactants interaction.

\title{
1 Introduction
}

Xanthan Gum (XG) is a high molecular weight polysaccharide produced by the bacterium xanthomonas campestris. It is produced commercially in a fermentation process. The XG molecule has a cellulosic backbone with side chains that cover the backbone and protect it across wide $\mathrm{pH}$ ranges and salt concentrations. The rigid cellulosic backbone maintains the viscosity of 
solutions during heating [1]. Its ability to increase the viscosity even at low concentration has made it a suitable viscosifier and stabilizer. XG has currently a wide variety of industrial applications including salad dressings, syrups, dairy products, pharmaceuticals, and is applied in agriculture, enhanced oil recovery, drilling, etc. [2]. Bulk rheology properties of XG has been investigated by many researchers $[1,3,4]$. XG shows a very high viscosity at low shear rates but its resistance to flow decreases by increasing the shear rate. This shear thinning nature makes it suitable for drilling purposes in petroleum industry [2].

The effect of XG on surface tension of aqueous solutions has also been studied. Muthamizhi et al reported an increasing surface tension for XG solution by increasing XG concentration, though they reported a value of about $79 \mathrm{mN} / \mathrm{m}$ for $0.6 \%$ weight $\mathrm{XG}$ concentration which seems in contrast to other reported measurements. They used the drop weight method for surface tension measurements [6]. Lee et al measured surface tension of XG solution together with some other biopolymers using du Nouy ring method and drop weight method. They observed an increasing trend for surface tension versus polymer concentration for the du Nouy ring method while the drop weight method showed an opposite trend. They concluded that the surface tension values of biopolymers depend on the measurement technique [7]. Khan et al stated that the surface tension of mixed solutions of XG and SDS decreases with XG concentration increase. However, they did not report a distinct trend for the XG-SDS interaction and related this surface tension decrease to the fact that $\mathrm{XG}$ itself reduces the surface tension in the aqueous solution in absence of any surfactant [5].

Despite some experimental investigations, to the best of our knowledge, the dynamic surface behavior of XG solution has not been investigated yet using an acceptable method. In this work, this issue was investigated using profile analysis tensiometry (PAT). In addition, the thixotropy 
effect of XG on the dynamic surface tension behavior was discussed and the interactions of XG SDS and XG - Triton X100 mixtures was studied experimentally.

\section{Experimental setup and procedure}

The surface tension and elasticity measurements were conducted using PAT manufactured by Sinterface Technologies (Berlin, Germany). The details on this device could be found in [8]. For performing each experiment, a drop was formed in a closed cuvette by injecting fluid using the syringe pump until a desired drop size was achieved, afterwards the surface tension was recorded versus time by keeping the drop size constant until 600 seconds and then, drop volume oscillations with frequencies of $0.02 / \mathrm{s}, 0.05 / \mathrm{s}$ and $0.08 / \mathrm{s}$ were carried out.

Fig. 1-A shows the results of numerical solution of Gauss Laplace set of equations via software. In PAT, the value of surface tension is calculated by tuning the result of numerical solution to the experimental drop profile. When we have Laplacian shapes, the error should be less than $1 \mu \mathrm{m}$ and distributed evenly over the profile. Fig. 1-B also depicts the procedure for elasticity measurements.

The buoyancy force measurements of XG solutions were performed using an Attension Sigma700 apparatus. This device measures the buoyancy force exerted by the fluid to a spherical probe with the resolution of $0.1 \mu \mathrm{N}$ (i.e. $0.01 \mathrm{mg}$ weighting resolution).

XG and Triton X100 were purchased from Sigma Aldrich and SDS was provided from AppliChem. All the experiments were performed at constant temperature of $22^{\circ} \mathrm{C}$. All aqueous solutions were prepared using deionized water. 


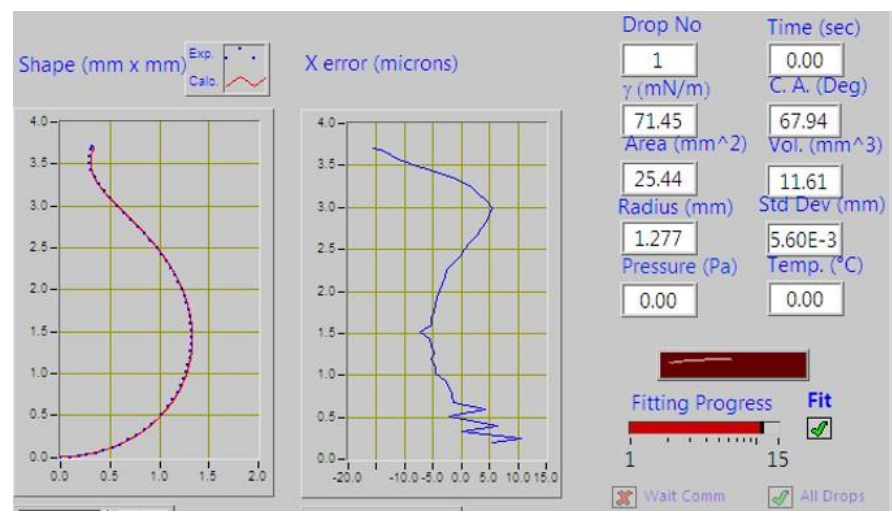

$\mathbf{A}$

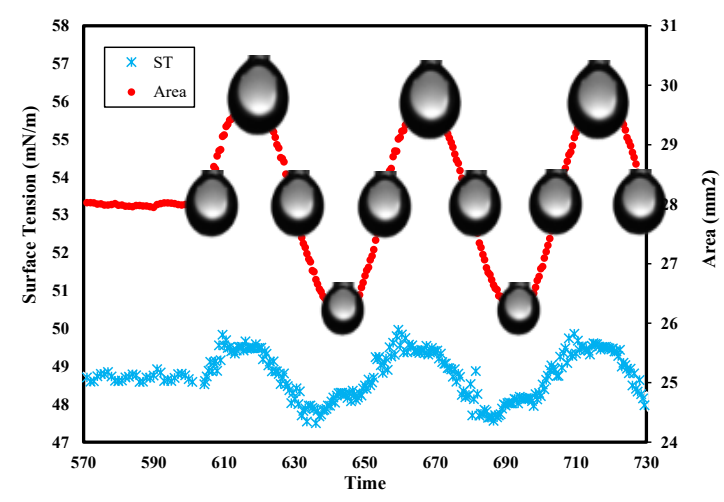

B

Fig. 1. Drop profile Analysis Tensiometry (PAT, SINTERFACE) for dynamic surface tension and interfacial elasticity measurements (via an oscillating drop protocol, right)

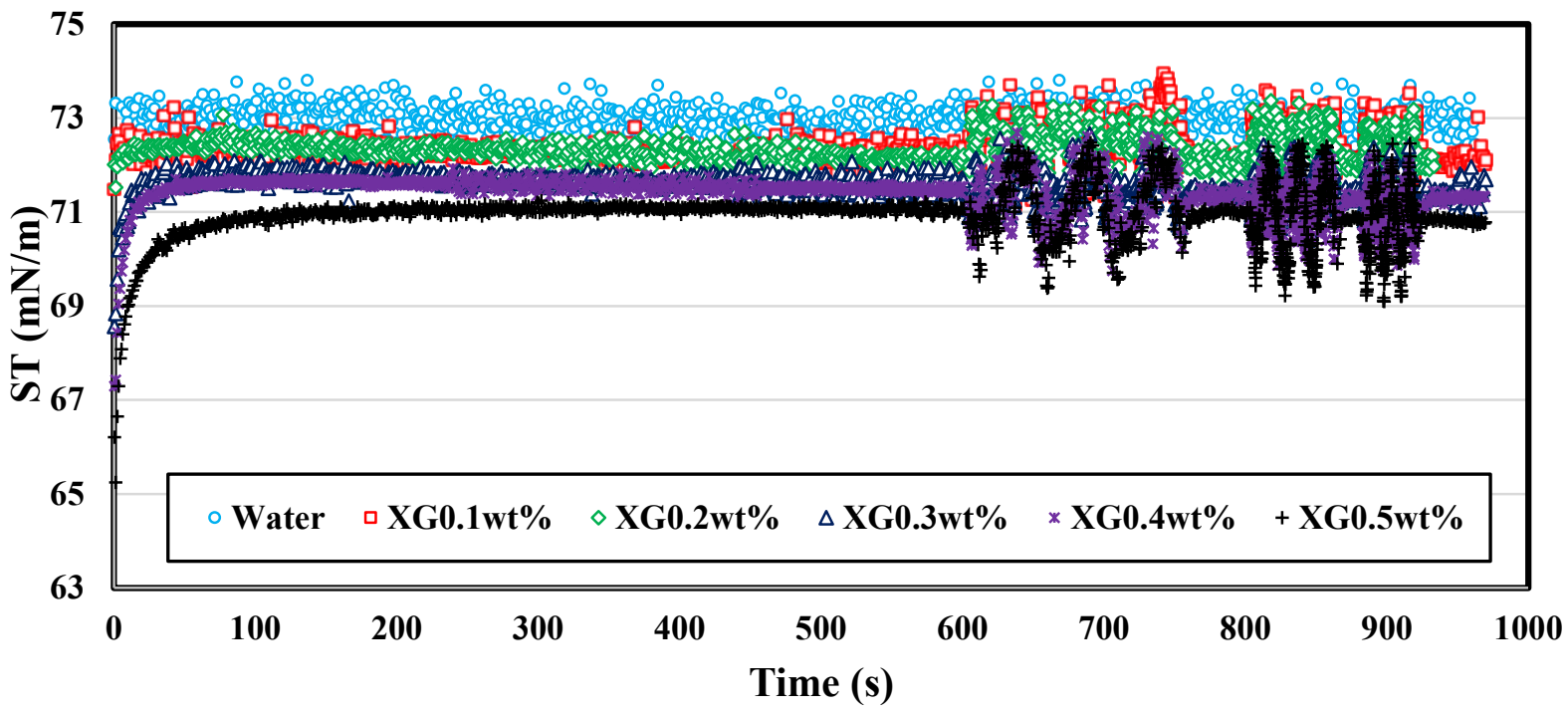

Fig. 2. Dynamic surface tension of $X G$ solution versus time for different concentrations of $X G$ 


\section{Results and discussion}

\subsection{Dynamic surface tension data of XG solution}

Fig. 2 depicts the dynamic surface tension of aqueous XG solutions for different concentrations of XG. According to the figure, the surface tension of the XG solution starts at a value less than equilibrium surface tension, then increases with time until reaching a plateau. The equilibrium surface tension values are close to that of pure water for different XG concentrations which shows weak surface activity for XG molecules.

Now, the question arises: why does the surface tension of XG solutions increase with time? One may think that XG molecules are present at the surface at the beginning of drop formation and leave the surface gradually toward the bulk. But this hypothesis cannot be true because it takes long times for large molecules such as XG to diffuse and adsorb at the drop surface. Moreover, the surface elasticity measurements at the beginning of the experiments showed approximately the same elasticity values as later measurements at equilibrium condition which shows approximately no $\mathrm{XG}$ is transferred from the bulk to the surface or back.

Therefore, this observation must be caused by a different mechanism, may be by bulk properties. This argument was supported by buoyancy force measurements using the densitometer Sigma 700. In these experiments, a spherical probe was immersed into the XG solution with a speed of $20 \mathrm{~mm} / \mathrm{s}$ and was stopped at the depth of $15 \mathrm{~mm}$, afterwards the buoyancy force of the fluid was recorded versus time. Fig. 3 shows a schematic of the buoyancy force measurements system.

Fig. 4 shows buoyancy force changes of aqueous XG solutions versus time for different XG concentrations. The buoyancy force reduces with time and the changes are larger for higher XG concentrations. The results shown in Fig. 4 reveal the existence of a time dependent force in the 


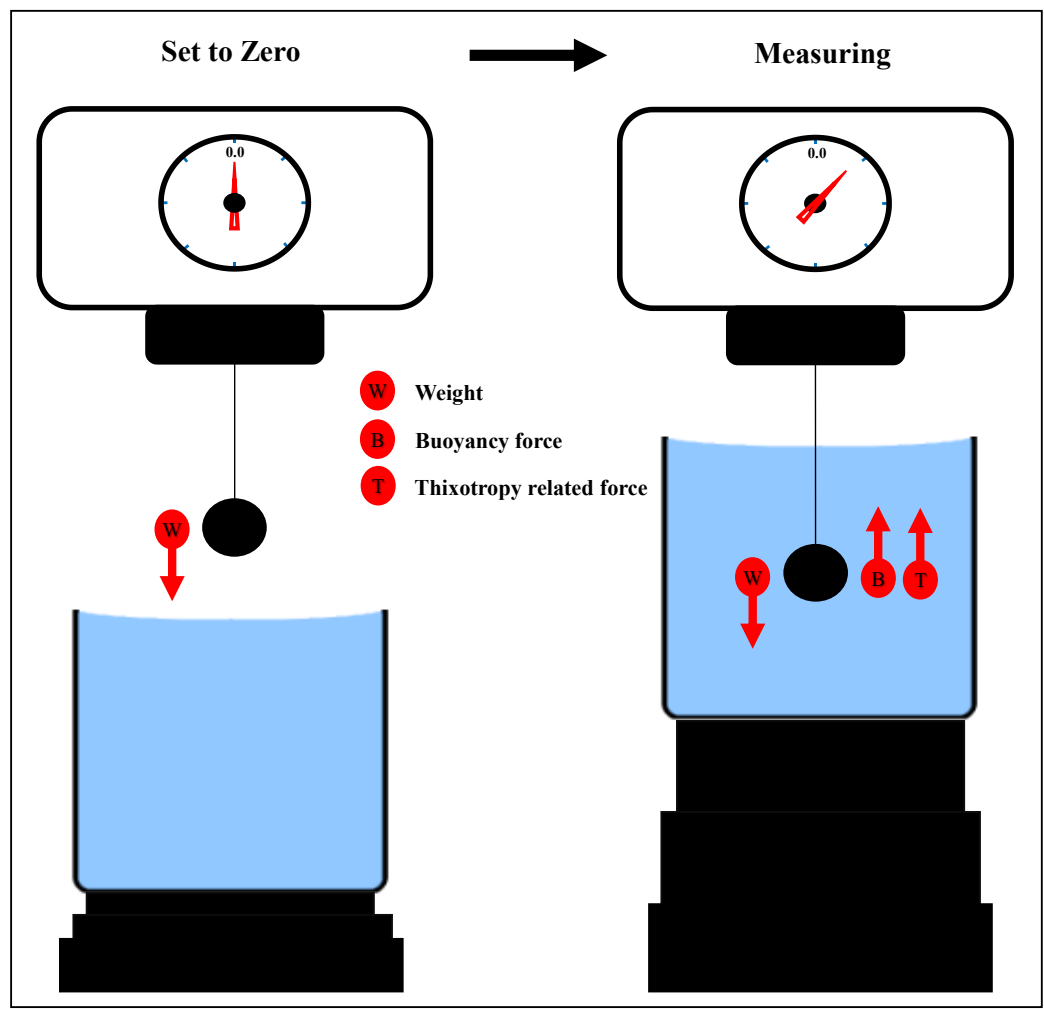

Fig. 3. Schematic buoyancy force/density measurement system

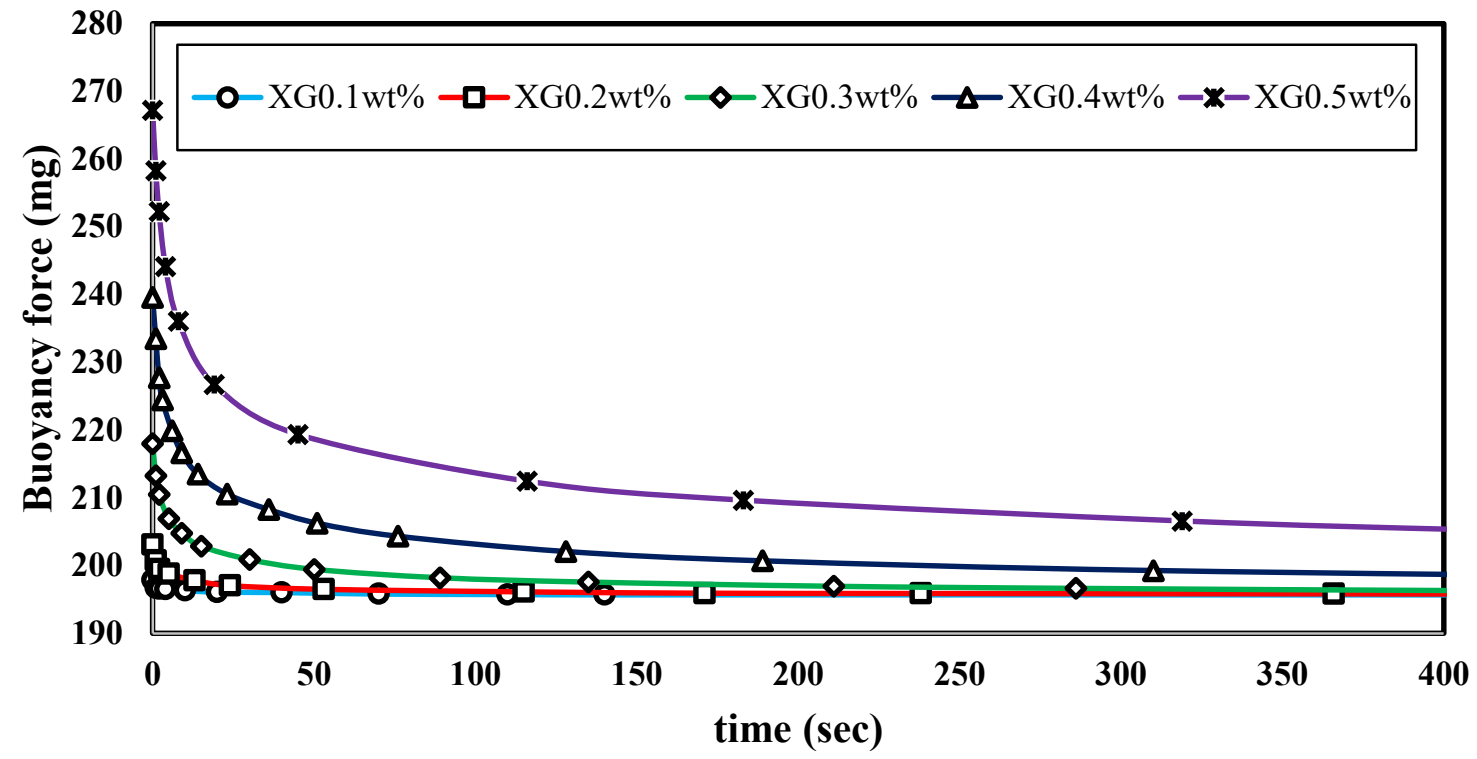

Fig. 4. Buoyancy force vs. time for different concentrations of XG 
XG solution. Existence of this force can be the main reason for the observed increasing surface tension of the XG solution with time.

Another experiment was conducted in which the spherical probe was immersed in the fluid until the depth of $30 \mathrm{~mm}$, then it was gradually moved upward and was stopped at the depth of $15 \mathrm{~mm}$. The results are depicted in Fig. 5. This figure shows an opposite behavior for buoyancy force measurements after downward and upward probe movements. Thus, it can be concluded that the direction of the measured force is opposite to the probe movement direction. According to the direction dependent nature of this force, one can conclude that it is not in relationship with fluid bulk density. It is also worth to mention that the density of each XG solution was separately measured by weighting some predetermined volume of the fluid and no changes versus time was observed. Besides, a density close to that of water was calculated for each case with a slight increase with the XG concentration.

For better understanding of this phenomenon, we should note that the quasi-static condition after stopping the macroscopic movement does not mean a full static condition in microscopic scale nearby the solid sphere due to rearrangements of the polymer molecules. In rheological experiments, which are macroscopic, the mentioned rearrangements after each change of shear rate cause a continuous change in viscosity. Here the molecular rearrangements after stop of the bulk movement, cause a micro scale local relaxation force which is evaluated via buoyancy force measurements.

The concept of thixotropy may justify this behavior. Aqueous XG solutions are widely known as thixotropic fluids [10]. In these fluids, the shear stress changes with time at constant shear rate. In addition, when the fluid is stopped, it takes some time for the shear stress to reach zero. In 


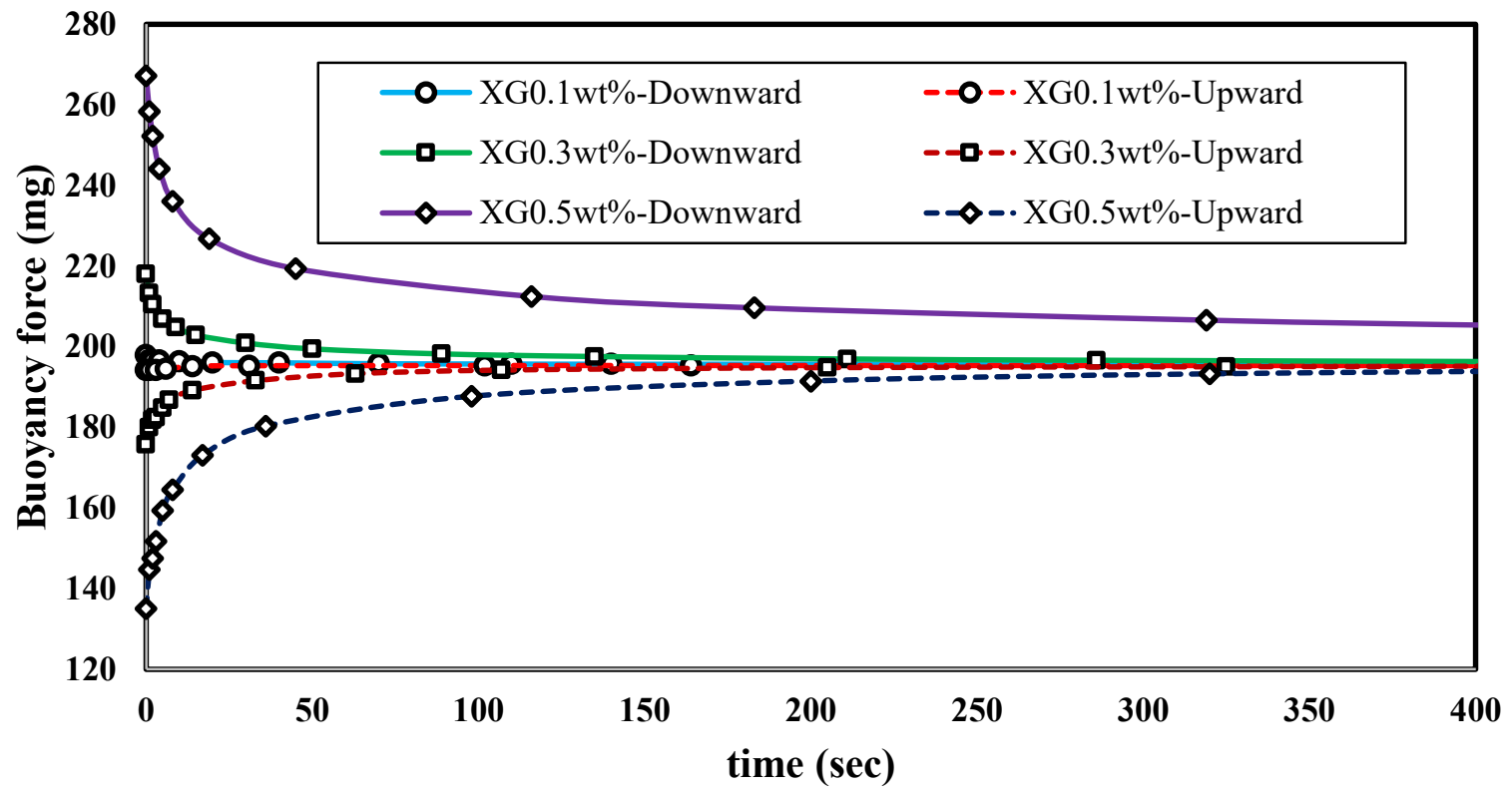

Fig. 5. Buoyancy force of different XG solutions after downward and upwards applied shear 
rheological literature, thixotropy is defined as the continuous decrease of viscosity with time when flow is applied to a sample that has been previously at rest and the subsequent recovery of viscosity in time when flow is discontinued [9]. When the flow is discontinued, microscopic movement and rotation of molecules is still going on until they rearrange in a three-dimensional structure due to the new condition [10]. In rheological experiments, the mentioned rearrangements after each change in shear rate induce a continuous change in viscosity. However, here we have measured these molecular rearrangements using a buoyancy force measurement device. Fig. 6 schematically depicts this re-structuring behavior. Since XG molecules bear negative charge, they tend to repel each other at rest and get optimum distance from each other. However, when a flow/shear is applied to the system, XG molecules are forced to get directed according to the flow/shear applied. When the applied flow/shear is discontinued, the negative charges dominate again and force gradually the XG molecules to rearrange to the rest condition that can cause a microscale relaxation force in opposite direction of the movements.

By connecting the concept of thixotropy to our problem, it could be said that the mentioned time dependent force is developed in the system because of the molecules movement and rotation after the flow/shear is stopped. This force has two specifications; first it decreases with time until it reaches zero and second, the direction of this force is related to the direction of the probe movement. This causes two almost symmetrical behaviors for buoyancy force changes in the case of upward and downward probe movement, as it is depicted in Fig. 5. In addition, similar to the thixotropy concept which is shear dependent, the mentioned force is affected by the velocity of the spherical probe movement as shown in Fig. 7. 


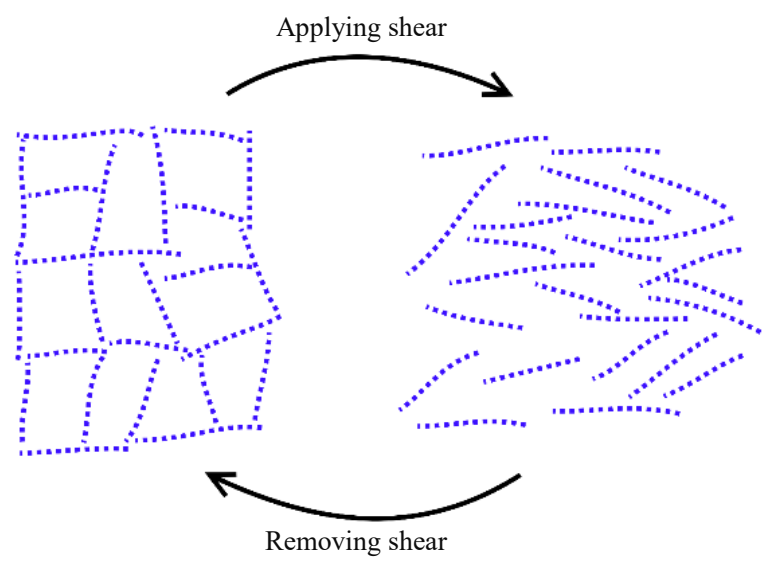

Fig. 6. Changes of a molecular structure by applying and removing shear

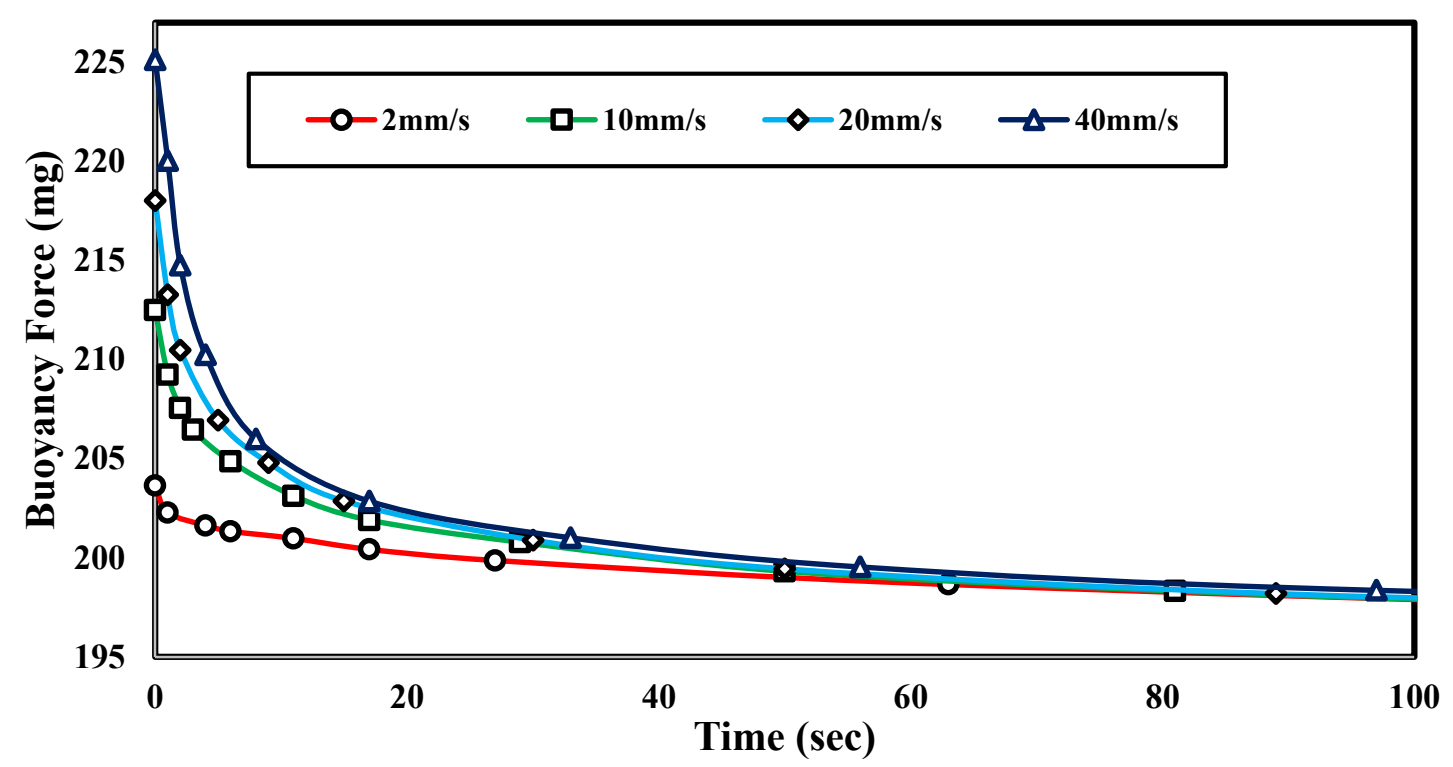

Fig. 7. Buoyancy force changes vs. time for different probe movement speeds for $0.3 \mathrm{wt} \% \mathrm{XG}$ solution. 
Now, we should consider that a time dependent force is present during the surface tension measurements with the PAT method. The existence of this force unbalances the balance between gravity and surface forces for a period of time until the molecules are re-structured according to the shear applied during drop formation. The force applied due to the fluid thixotropic behavior must be added to the Gauss Laplace Equation to improve surface tension predictions [11, 12].

\subsection{XG interaction with SDS surfactant}

Let us now discuss the interaction between XG and SDS molecules. The dynamic surface tension behavior of mixed aqueous XG-SDS solutions was studied using PAT. As an example, the results of experiments for the case of $0.25 \mathrm{cmc}$ SDS and different $\mathrm{XG}$ concentrations are depicted in Fig. 8. The increasing surface tension behavior of XG solutions (as discussed in the previous section due to thixotropic effects) has affected the dynamic surface tension behavior of mixed solutions. There is a competition between SDS adsorption and XG thixotropy behaviors. According to Fig. 8, at lower concentrations of XG, the dynamic behavior is decreasing. This is due to the fact that SDS molecules diffuse from the bulk, adsorb at the surface and reduce the surface tension. But at higher concentrations of $\mathrm{XG}$ at which the thixotropy effect becomes stronger, the dynamic surface tension shows an increasing behavior with a shallow maximum until the respective plateau value is reached.

The equilibrium surface tension data measured for mixed XG-SDS solutions with different concentrations are given in Fig. 9. This figure shows a strong interaction between XG and SDS molecules. The equilibrium surface tension of XG aqueous solution, in absence of SDS, slightly decreases with XG concentration but the changes are less than $2 \mathrm{mN} / \mathrm{m}$. However, in presence of SDS, this decrease is much larger. For example, for cases of $0.25 \mathrm{cmc}$ and $0.5 \mathrm{cmc}$ SDS concentrations, the variation of XG concentration from 0 to $0.5 \mathrm{wt} \%$ has caused about $13 \mathrm{mN} / \mathrm{m}$ 


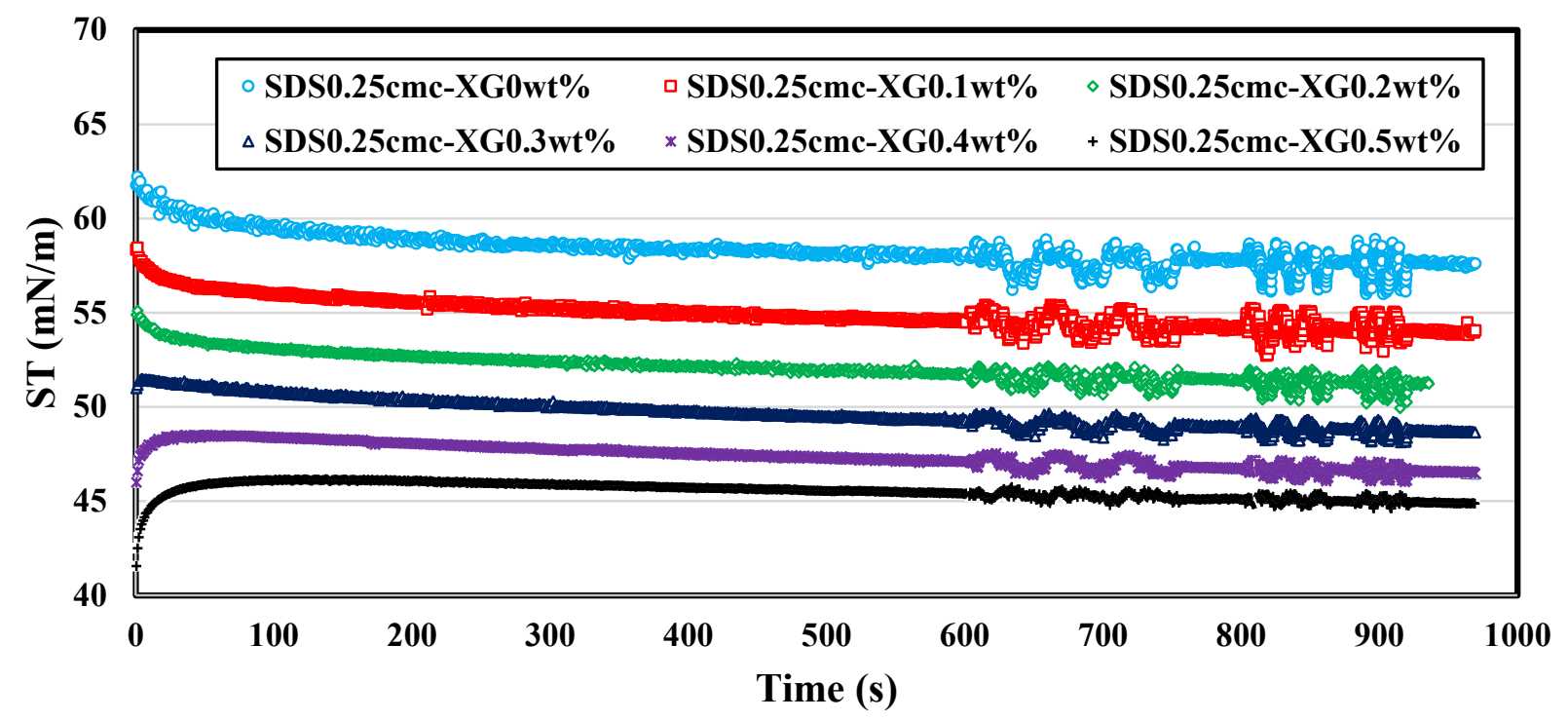

Fig. 8. Dynamic surface tension of $0.25 \mathrm{cmc}$ SDS solution with different $X G$ concentrations

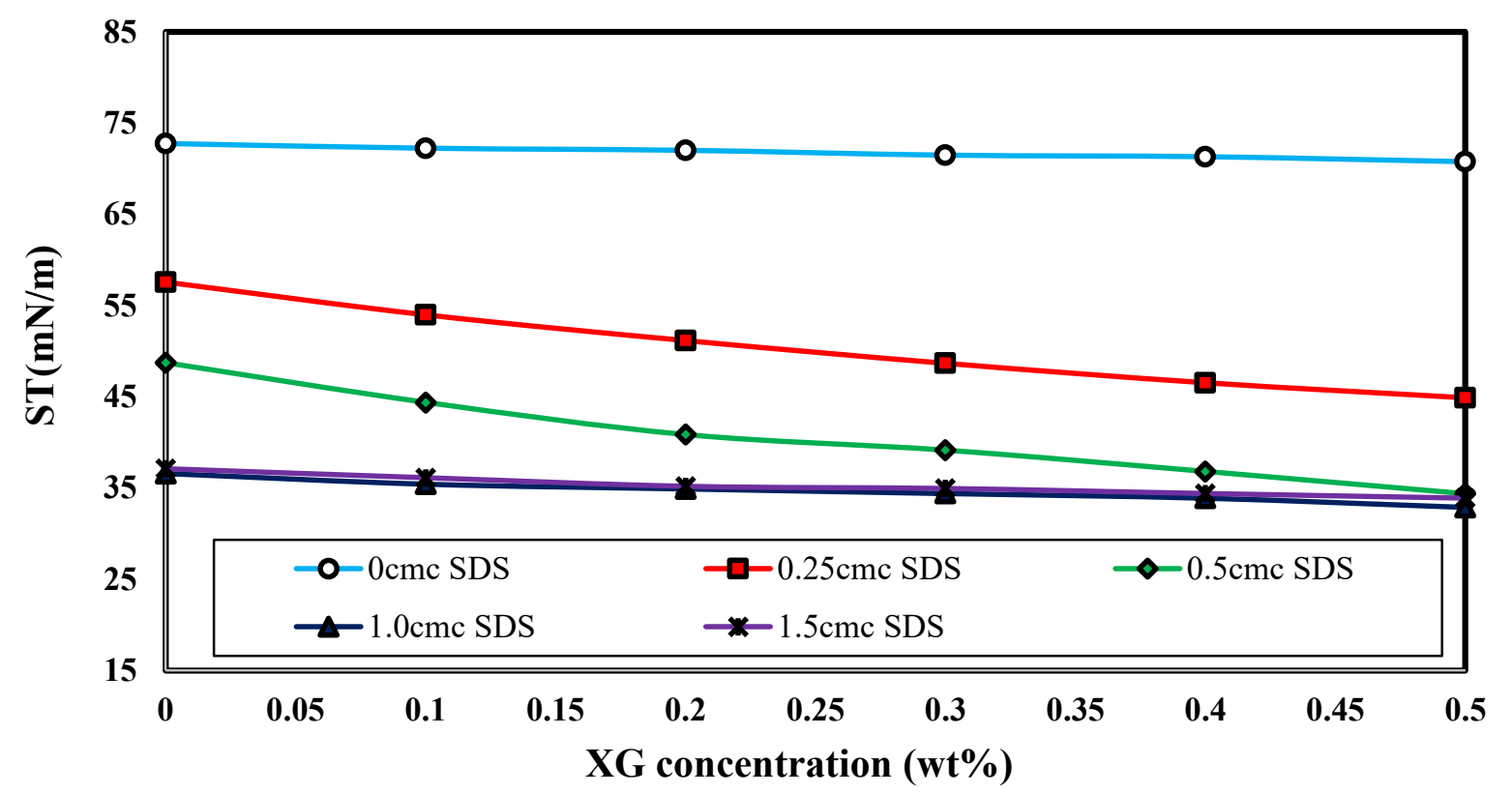

Fig. 9. Equilibrium surface tension of mixed SDS / XG solutions for different concentrations of SDS and 
and $15 \mathrm{mN} / \mathrm{m}$ reduction in the surface tension, respectively. This may be justified by the anionic nature of XG molecules which repel SDS molecules from the bulk toward the surface. Moreover, for the case of $0.5 \mathrm{cmc}$ SDS concentration, the equilibrium surface tension reduces by increasing $\mathrm{XG}$ concentration to a value of $34.37 \mathrm{mN} / \mathrm{m}$ which is close to the measured surface tension at CMC. This implies that we can reach to lower values of surface tension without enhancing the surfactant concentration, i.e. by adding some $\mathrm{XG}$ to the solution. This is very important in polymer-surfactant flooding processes in which we need to increase the viscosity and decrease surface tension at the same time [13].

\subsection{Interaction between XG and Triton X100}

Similar experiments as with mixed XG and SDS solutions were repeated using mixed solutions of XG and Triton X100 to investigate the possible interactions between XG and a nonionic surfactant. As an example, Fig. 10 depicts the dynamic surface tension measurements of XGtriton X100 aqueous solutions for the case of $0.25 \mathrm{cmc}$ triton $\mathrm{x} 100$ concentration.

According to this figure, the dynamic surface tension of XG-Triton X100 is always decreasing. This is because the concentration of Triton X100 is of the order of $10^{-4} \mathrm{mM}$. At this range of concentration, it takes hundreds of seconds for Triton X100 molecules to reach the surface and reduce the surface tension. Because of this long-term dynamic behavior, the influence of the XG thixotropic behavior cannot be observed for the equilibrium surface tension of Triton X100 solutions. This is why the results of equilibrium surface tension for the pair of XG-Triton X100 in aqueous solution show no significant synergy between XG and Triton X100 molecules. In another word, the surface tension values are strongly dependent on the Triton concentration while showing a weak dependency on the XG concentration. However for oscillating drop protocol, due to dynamic conditions 


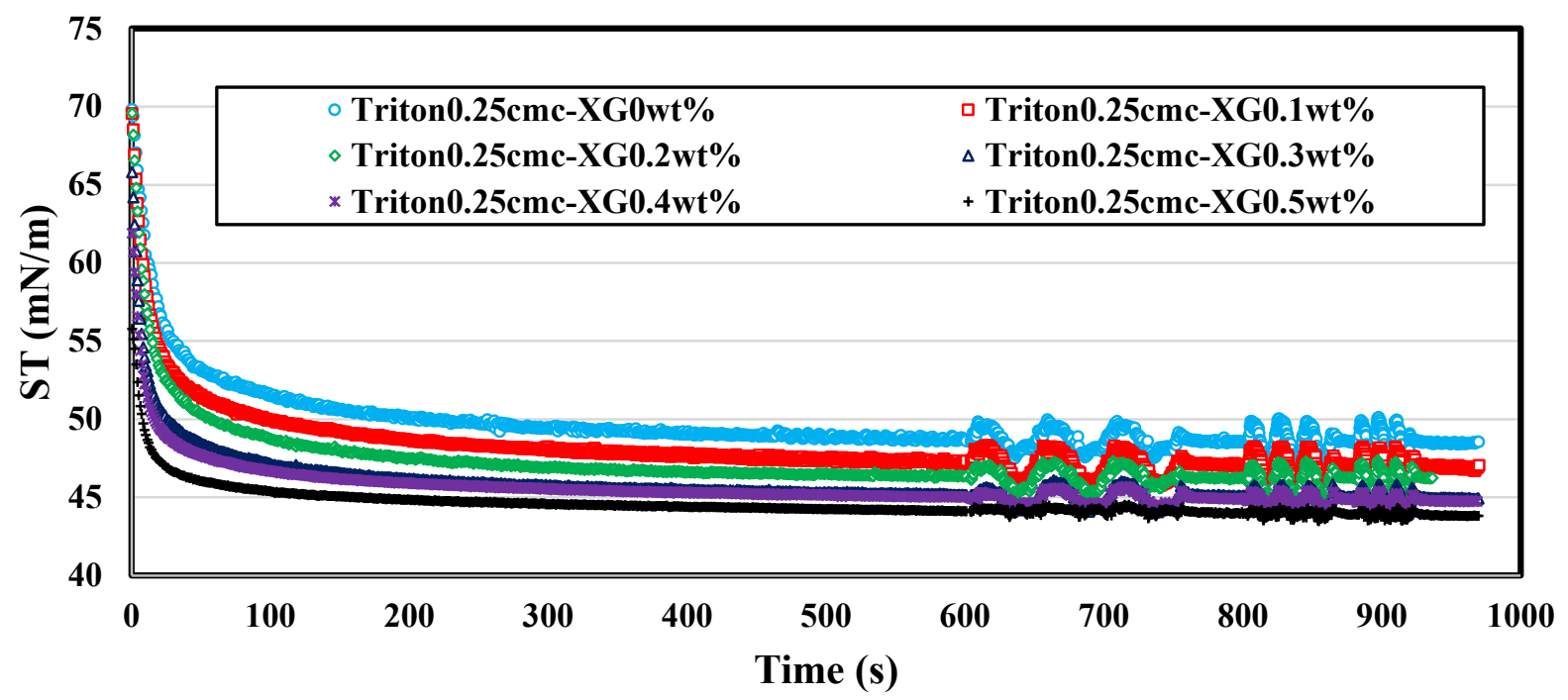

Fig. 10. Dynamic surface tension of $0.25 \mathrm{cmc}$ triton $X 100$ solution with different $X G$ concentrations

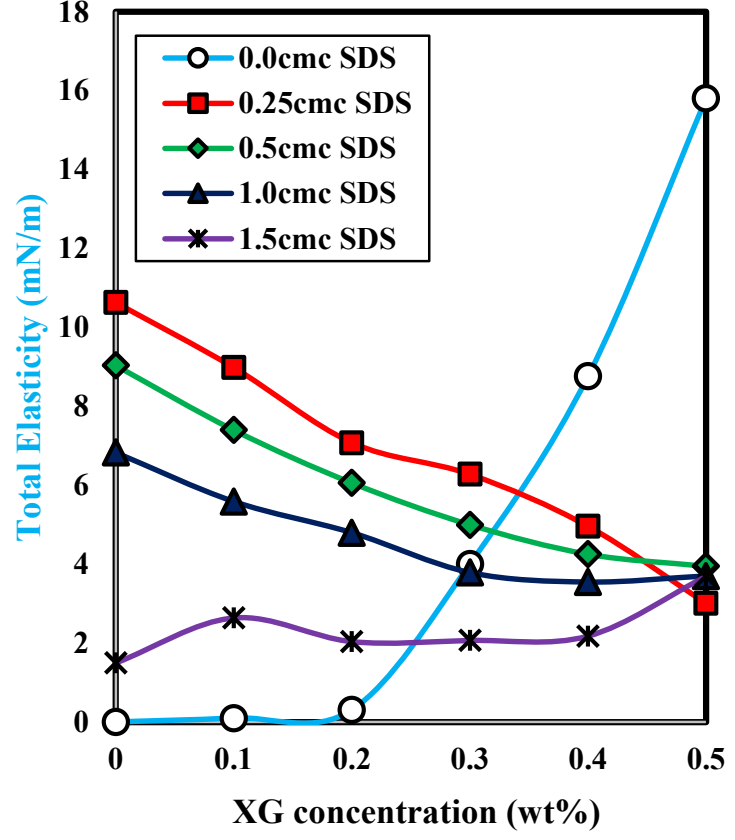

A) XG-SDS

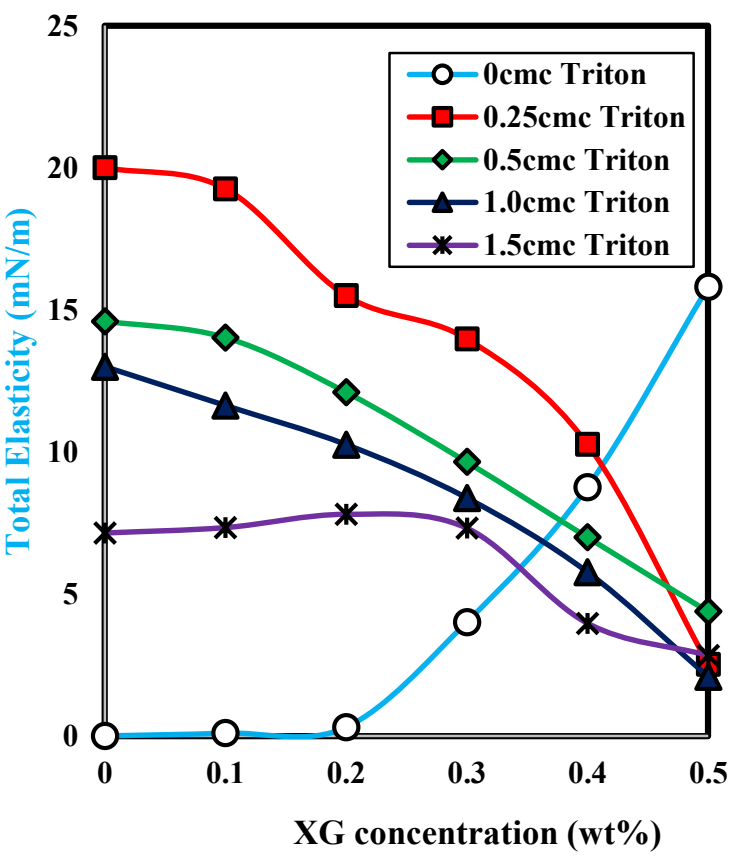

B) XG-Triton X100

Fig. 11. Combined surface and thixotropic elasticity values for different concentrations 


\subsection{Elasticity calculations}

The results of elasticity measurements are depicted in Fig. 11. According to these figures, for the case of pure XG solutions, the elasticity increases with increasing XG concentration. According to the mentioned results in this work, as we do not expect a real adsorbed layer of XG at the drop surface, and no surface tension changes were observed for quasi-static and equilibrium conditions, this apparent elasticity is due to the fluid thixotropic behavior that influences the drop profile. Then for an efficient discussion on the two mentioned simultaneous effects influencing the drop profile with different mechanisms, measured here via drop oscillations, the termini of interfacial and thixotropic elasticities are used here.

Fig. 12-A shows the elasticity behavior for $0.5 \mathrm{wt} \% \mathrm{XG}$ aqueous solution in which the surface tension changes are in the opposite direction of the drop area changes while in Fig. 12-B, which shows the surface tension data of Triton X100 0.25 CMC aqueous solution, the surface tension changes are in the same direction as drop area changes. Fig. 12-B shows the adsorption layer elasticity behavior. In this behavior, by diminishing the surface area, since the extra surfactants cannot leave the surface promptly, the amount of surfactants per unit area increases and the surface tension is reduced. The opposite occurs when surface area is enlarged. However, Fig. 12A shows an apparent surface elasticity which is at the opposite direction of area changes and, as discussed in section 3.1, is due to the thixotropic behavior of XG. In other words, the thixotropic and adsorb layer elasticities show respectively negative and positive viscous modules. This is because the phase angle of these two behaviors differs and combining them provides a system at which two opposite elasticity behaviors act against each other and can be compensated. This behavior can be observed in Fig. 12-C. 


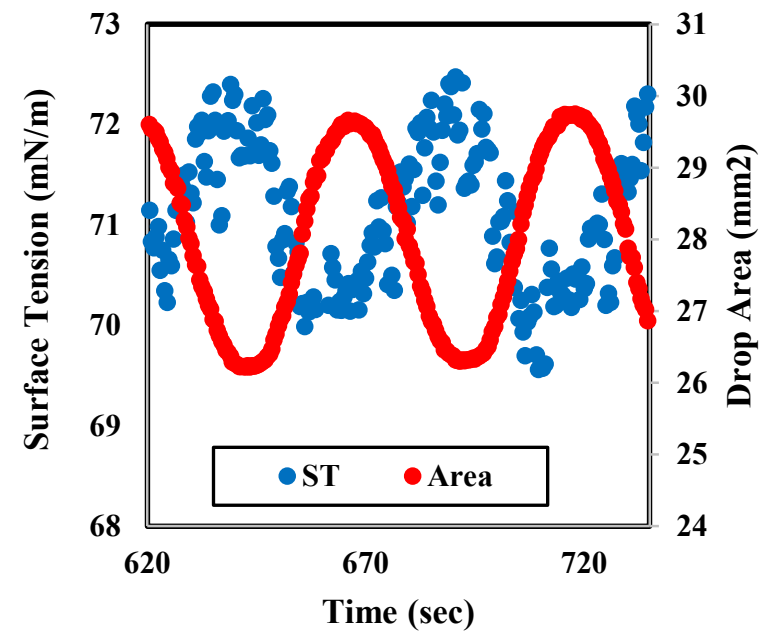

A) Thixotropic elasticity

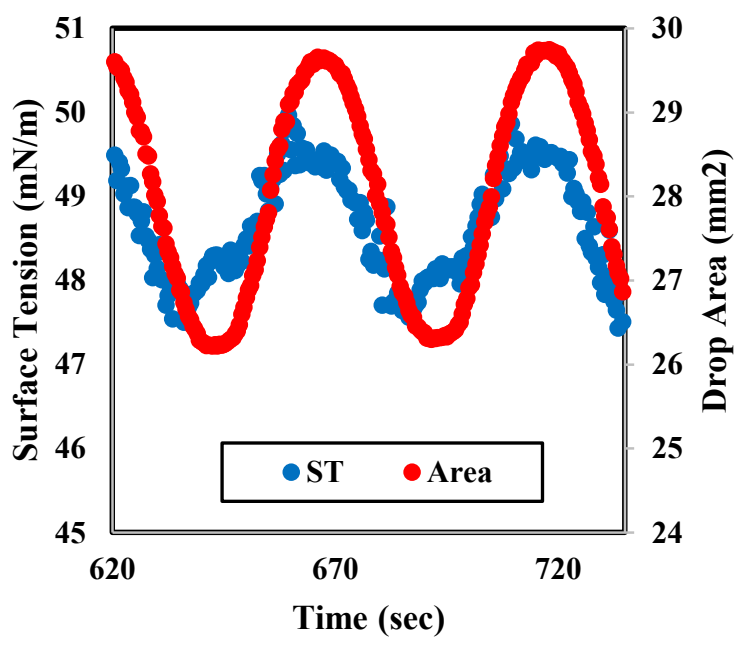

B) Adsorption layer elasticity

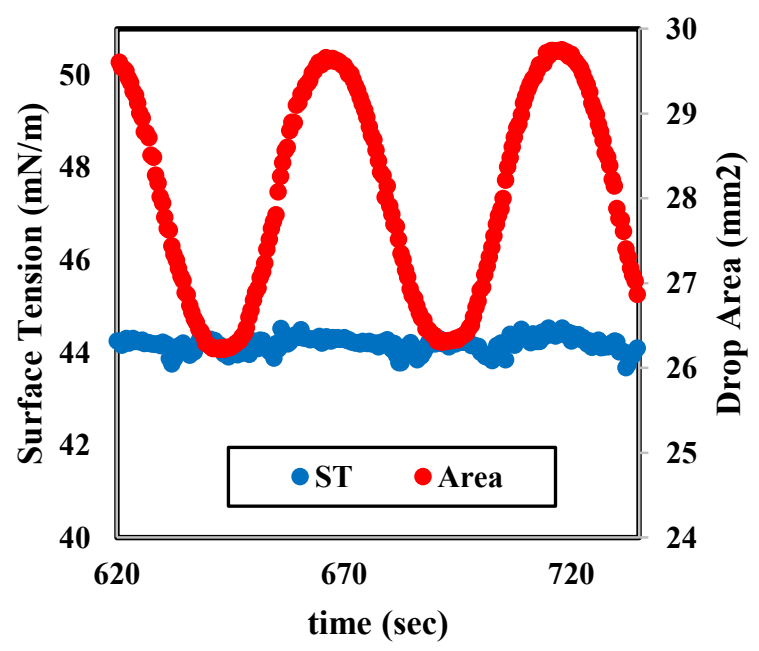

C) Combined thixotropic and adsorption layer elasticity

Fig. 12. Surface tension and drop area changes vs. time for cases of pure A) thixotropic elasticity, B) adsorption layer elasticity and C) combined thixotropic and adsorption layer elasticity behaviors 
Therefore, when both XG and either surfactants of SDS and Triton X100 are present in the solution, since the thixotropy induced elasticity is at the opposite direction of adsorption layer elasticity, by increasing XG concentration, the thixotropy induced elasticity increases and compensates the adsorption layer elasticity. In addition, for the case of SDS, by increasing XG concentration, more repulsive force is exerted to the SDS molecules by XG molecules pushing them toward the surface. This enhances the SDS concentration at the surface and in the subsurface region. At this condition, the low frequency surface elasticities will be decreased because the changes in area are compensated easily by the available surfactant molecules.

However, for the case of $1.5 \mathrm{cmc}$ SDS concentration, an almost constant surface elasticity versus XG concentration is observed. In this case, the SDS concentration at the surface has reached a maximum value which keeps surface elasticity at a low level. It also should be mentioned that the thixotropic behavior of $\mathrm{XG}$ solutions is weakened by surfactant concentration which influences the surface elasticity values (Fig. 13).

\section{Conclusions}

Dynamic surface tension and interfacial elasticity of aqueous XG solutions show abnormal results (increasing surface tension with time) which are explained by the thixotropic properties of the polymer solution. XG/surfactants mixed solutions are also studied and the following conclusions can be drawn:

- A new method based on accurate buoyancy force measurements of a falling/rising solid sphere in a polymer solution is introduced. 


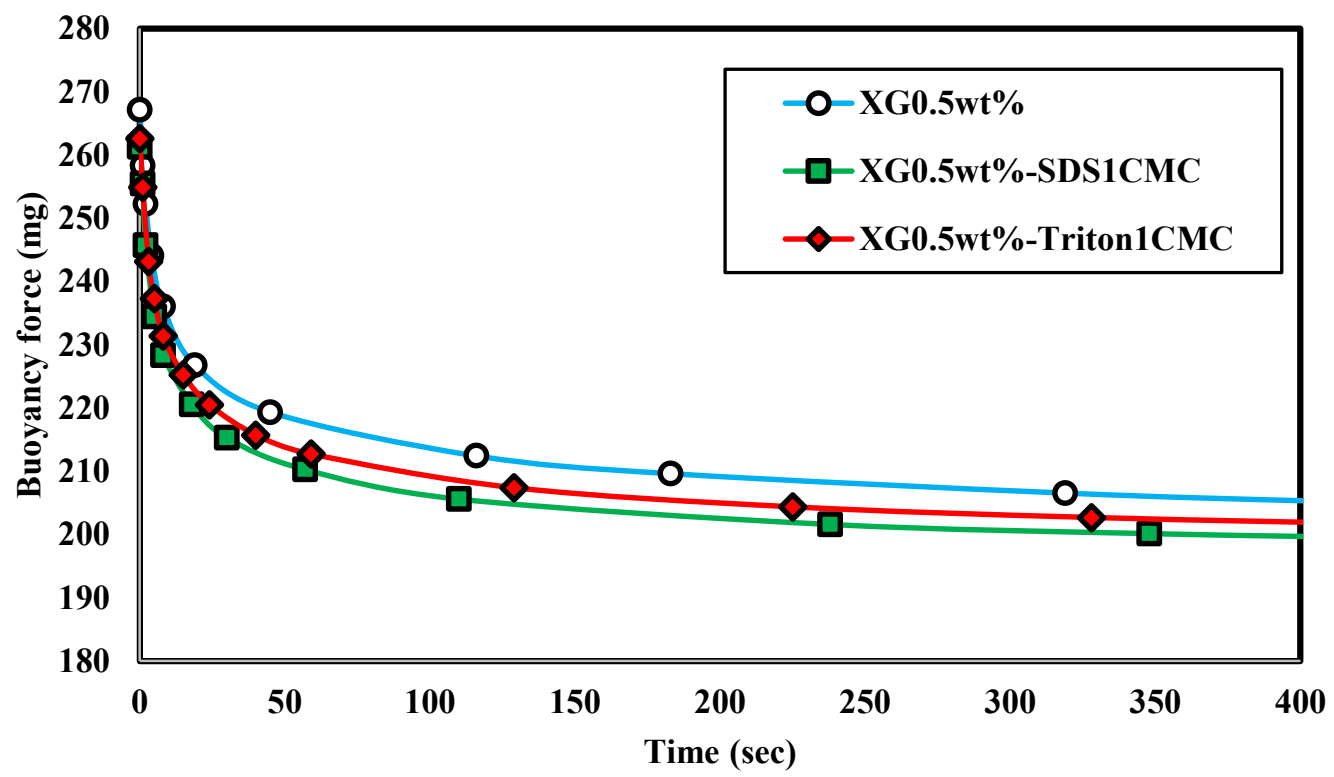

Fig. 13. Buoyancy force changes vs. time for aqueous solutions of XG with and without SDS and Triton X100 surfactants 
- Profile analysis tensiometry used in the drop oscillation protocol can be introduced as a novel high sensitive technique for measurements of polymer solution's thixotropic properties. The thixotropic elasticity values which are against the interfacial elasticity values typically measured for surfactant solutions by the same technique can be measured also separately and discussed meaningfully.

- As the thixotropic effects are a function of polymer concentration and the forced shear rate (depends on fluid movement degree) during dynamic surface tension measurements of XG-SDS mixed solutions, the competition between XG thixotropic bulk elasticity and SDS dynamic of adsorption and elasticity can create different situations, however, all are understandable and can be interpreted well, considering results of single XG solution.

- For the case of Triton X100, since the dynamics of adsorption is slower and the surfactant is also nonionic with negligible interaction with $\mathrm{XG}$, a regular dynamic behavior is observed (i.e. decreasing surface tension), however one should consider that this dynamic is also changed due to polymer thixotropic effect in the background.

- There exists a strong interaction between XG and SDS molecules which could not be observed for XG and Triton X100. This strong interaction can be related to the anionic nature of both XG and SDS molecules. 


\section{References}

1 Sworn, Graham. "Xanthan gum." Food stabilisers, thickeners and gelling agents (2009): 325-342.

2 Garcia-Ochoa, F., V. E. Santos, J. A. Casas, and E. Gomez. "Xanthan gum: production, recovery, and properties." Biotechnology advances 18, no. 7 (2000): 549-579.

3 Whitcomb, P. J., and C. W. Macosko. "Rheology of xanthan gum." Journal of Rheology 22, no. 5 (1978): 493-505.

4 Zhong, Lirong, Martinus Oostrom, Michael J. Truex, Vincent R. Vermeul, and James E. Szecsody. "Rheological behavior of xanthan gum solution related to shear thinning fluid delivery for subsurface remediation." Journal of hazardous materials 244 (2013): 160170.

5 Khan, Mohammad Yunus, Abhijit Samanta, Keka Ojha, and Ajay Mandal. "Interaction between aqueous solutions of polymer and surfactant and its effect on physicochemical properties." Asia-Pacific Journal of Chemical Engineering 3, no. 5 (2008): 579-585.

6 Muthamizhi, K., P. Kalaichelvi, Shubhangi Tukaram Powar, and R. Jaishree. "Investigation and modelling of surface tension of power-law fluids." RSC Advances 4, no. 19 (2014): 9771-9776.

7 Lee, Boon-Beng, Eng-Seng Chan, Pogaku Ravindra, and Tanveer Ahmad Khan. "Surface tension of viscous biopolymer solutions measured using the du Nouy ring method and the drop weight methods." Polymer bulletin 69, no. 4 (2012): 471-489

8 Karbaschi, M., D. Bastani, A. Javadi, V. I. Kovalchuk, N. M. Kovalchuk, A. V. Makievski, E. Bonaccurso, and R. Miller. "Drop profile analysis tensiometry under highly dynamic conditions." Colloids and Surfaces A: Physicochemical and Engineering Aspects 413 (2012): 292-297.

9 Mewis, J. and Wagner, N.J., 2009. Thixotropy. Advances in Colloid and Interface Science, 147, pp.214-227.

10 Barnes, H.A., 1997. Thixotropy - a review. Journal of Non-Newtonian fluid mechanics, 70(1-2), pp.1-33.

11 Adamson, A.W. and Gast, A.P., 1967. Physical chemistry of surfaces.

12 Berry, J.D., Neeson, M.J., Dagastine, R.R., Chan, D.Y. and Tabor, R.F., 2015. Measurement of surface and interfacial tension using pendant drop tensiometry. Journal of colloid and interface science, 454, pp.226-237.

13 Shah, D.O. ed., 2012. Improved oil recovery by surfactant and polymer flooding. Elsevier. 